\title{
Supporting Information for: Size-Programmed Synthesis of PbSe Quantum Dots via Secondary Phosphine Chalcogenides
}

Amanda Preske ${ }^{\dagger}$, Sean W. O’Neillł, Brett D. Swartz ${ }^{\dagger}$, Jin Liu ${ }^{\dagger}$, Oleg V. Prezhdo ${ }^{\|}$and Todd D. Krauss $^{\dagger, \S, *}$

$†$ Department of Chemistry, $¥$ Materials Science Program, and the §Institute of Optics, University of Rochester, Rochester, New York 14627, USA

IDepartment of Chemistry, University of Southern California, Los Angeles, California 90089, USA

\section{PbSe concentration procedure}

Measuring the $\mathrm{PbSe} \mathrm{QD}$ concentration during a reaction necessitates minor changes to the typical secondary phosphine selenide and $\mathrm{Pb}$ (oleate) $)_{2}$ procedure described in the main text. The reaction was verified to be stable (via absorbance and photoluminescence showing no evidence of etching) when the condenser was removed and exposed to air under high nitrogen flow. Precisely timed and frequent aliquots were withdrawn using a calibrated micropipette and quenched in $3 \mathrm{~mL}$ of cold toluene. Samples were not washed in order to retain product and UV-vis spectra were obtained as described in the Experimental Section.

Absorbance was used to calculate QD size and molar extinction coefficient by a previously reported method. ${ }^{1,2}$ Since the molar extinction coefficient scales with the nanocrystal concentration and not size at high photon energies, QD absorbance at $400 \mathrm{~nm}$ was used for calculations. Per Dai et al., ${ }^{1}$ QD diameter scales with the bandgap (which is determined by the wavelength of the first exciton peak) according to:

$$
\mathrm{d}=\frac{\lambda-143.75}{281.25}
$$

where the $\mathrm{d}$ is the diameter of the QD (nm) and $\lambda$ is the wavelength of the first exciton peak (nm). At high photon energies, the molar extinction coefficient, $\varepsilon$, is given by: ${ }^{2}$

$\varepsilon_{3.1 e V}=(0.0277 \pm 0.0005) d^{3} \mathrm{~cm}^{-1} / \mu M$

Using beer's Law, $A=\varepsilon b C$, where $A$ is the absorbance at $400 \mathrm{~nm}$, $b$ is the path length $(1 \mathrm{~cm})$ of the cuvette, and $\varepsilon$ is calculated as above, the concentration of the QD sample is determined. 


\section{5-Fold Scaled up reaction of DiBPSe and Lead Oleate}

To demonstrate sensible scale up, a smaller reaction volume (rather than increasing everything 22.5 -fold) was used. $\mathrm{Pb}$ (oleate) $)_{2}$ was created by combining $1.51 \mathrm{~g} \mathrm{PbO}(6.7 \mathrm{mmol})$ with $18.46 \mathrm{~mL}$ oleic acid $(58.5 \mathrm{mmol})$ and $89.5 \mathrm{~mL}$ decane in a $250 \mathrm{~mL}$ 3-neck flask. The contents were heated to $120{ }^{\circ} \mathrm{C}$ under $\mathrm{N}_{2}$ flow with a condenser and heated for 25 minutes to yield a clear, colorless solution of lead oleate. No purification was performed, and the as-produced $\mathrm{Pb}$ (oleate) $)_{2}$ was left in the flask and reduced to $75^{\circ} \mathrm{C} .4 .5 \mathrm{~mL}$ of $1 \mathrm{M}$ DiBPSe was injected and the solution instantly turned from clear and colorless to dark brown. The reaction proceeded for 4 minutes and was thermally quenched on an ice bath and with the addition of $100 \mathrm{~mL}$ of cold ethanol. The mixture was divided into separate Eppendorf tubes and centrifuged at 10,000 RPM for 10 minutes to yield solid, dark brown pellets with a clear supernatant. The supernatants were discarded and the pellets were redispersed in tetrachloroethylene and combined for characterization. An aliquot of known volume was withdrawn from the flask prior to quenching with ethanol in order to determine the yield.

\section{PbSe Reaction Yield}

The chemical reaction yield was calculated using the mean QD diameter, extinction coefficient, and QD concentration as detailed above. A known sample volume allowed determination of the sample QD concentration, which was then scaled to determine the number of QDs produced by a given synthesis. The $\mathrm{QD} \mathrm{Pb}$ :Se ratio and number of $\mathrm{Se}$ or $\mathrm{Pb}$ atoms per $\mathrm{QD}$ was estimated as a function of mean particle diameter based on data provided in Dai et al., which allowed calculation of the total amount of $\mathrm{Se}$ or $\mathrm{Pb}$ incorporated into QDs (i.e., mmol $\mathrm{Pb}$ and $\mathrm{Se}$ ). ${ }^{1}$ The ratio of the calculated quantity of $\mathrm{Se}$ and $\mathrm{Pb}$ incorporated into QDs relative to the known quantity of $\mathrm{Se}$ and $\mathrm{Pb}$ added during the synthesis is referred to as the reaction yield. 


\section{$\underline{\text { Precursor Conversion Kinetics }}$}

As demonstrated in previous work, the absorption at $\lambda=400 \mathrm{~nm}\left(\mathrm{~A}_{400 \mathrm{~nm}}\right)$ of a sample of colloidal $\mathrm{PbSe} \mathrm{QDs}$ is proportional to the concentration of crystalline PbSe units in solution ([PbSe]), and is independent of QD size. ${ }^{2,3}$ Therefore, $\mathrm{A}_{400 \mathrm{~nm}} \propto[\mathrm{PbSe}]$, and we used $\mathrm{A}_{400 \mathrm{~nm}}$ as a direct proxy for $\mathrm{PbSe}$ precursor coversion.

As detailed in the Experimental section of the main document, a series of aliquots were collected during synthesis of PbSe QDs. $\mathrm{A}_{400 \mathrm{~nm}}$ for these discrete time points were fit with an exponential function of the form $A_{400 \mathrm{~nm}}=A \cdot\left(1-e^{-k t}\right)$, where A is the curve amplitude (arbitrary units), $\mathrm{t}$ is the reaction time (s), and $\mathrm{k}$ is the precursor conversion rate constant $\left(\mathrm{s}^{-1}\right) . \mathrm{A}_{400 \mathrm{~nm}}$ were normalized such that the fit exponential curve converged to a limit of 1 (i.e., $\mathrm{A}=1$ ).

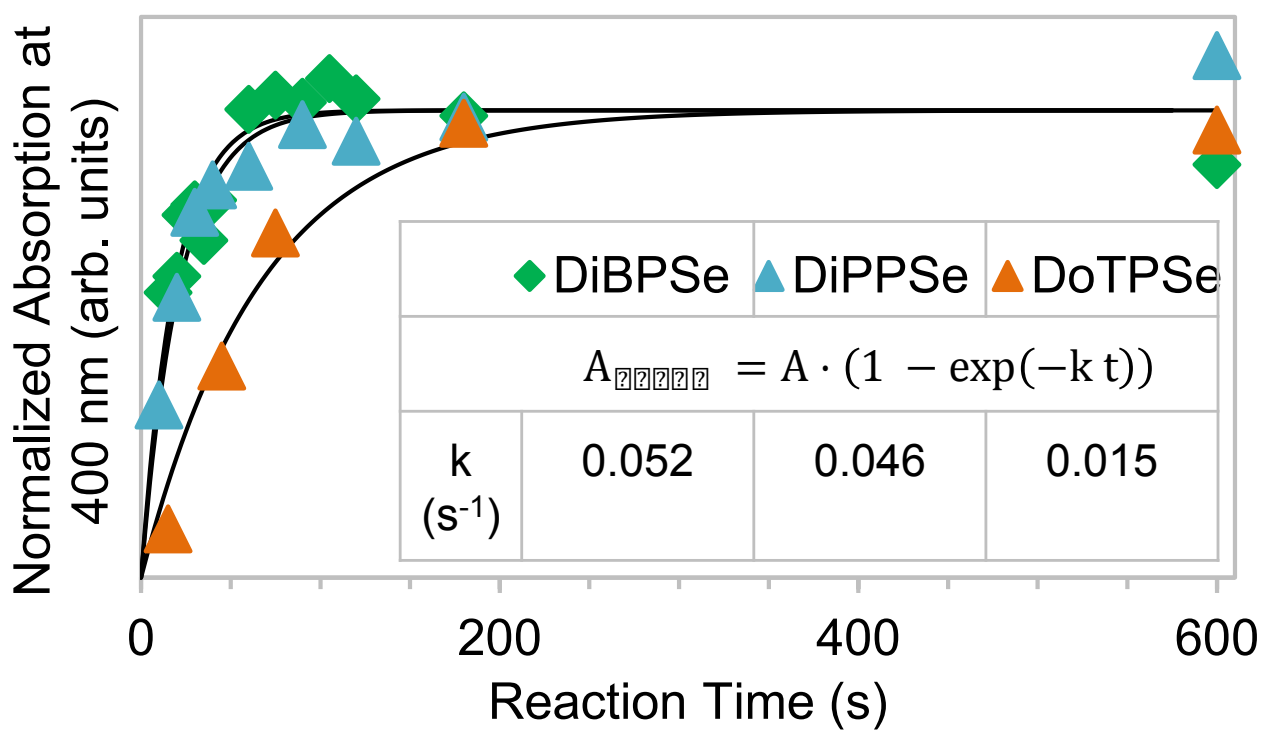

Figure S1. Rise in absorption at $400 \mathrm{~nm}\left(\mathrm{~A}_{400 \mathrm{~nm}}\right)$ over the course of QD syntheses using the secondary phosphine selenides DiBPSe, DiPPSe, and DoTPSe. Exponential fittings of the data were used to determine the precursor conversion rate constant, $\mathrm{k}$. 

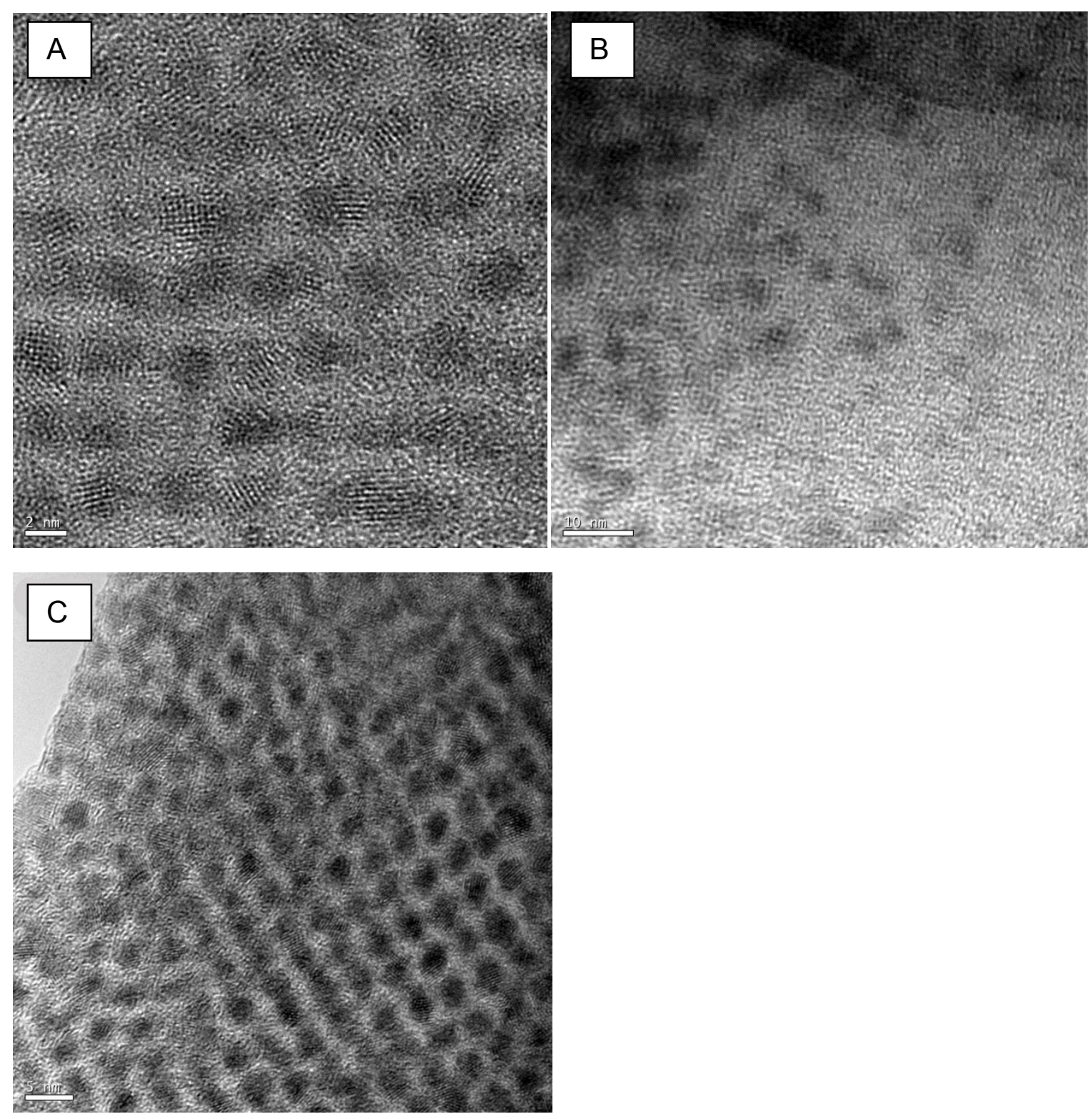

Figure S2. TEM Images of (a) DiBPSe, (b) DoTPSe, and (c) DEPSe-produced PbSe QDs upon reaction with lead oleate illustrate the different sizes obtainable under otherwise identical reaction conditions. Scale bars are (a) $2 \mathrm{~nm}$, (b) $10 \mathrm{~nm}$, and (c) $5 \mathrm{~nm}$, respectively. 
Table S1. Photoluminescence (PL) quantum yields (QY) and FWHM obtained for a series of PbSe quantum dots made with different secondary phosphine selenide precursors using the same precursor ratio $\left(1.0: 2.47: 1.0 \mathrm{Se}: \mathrm{Pb}(\mathrm{OA})_{2}\right.$ :excess $\left.\mathrm{OA}\right)$ decline with an increase in diameter. It was observed that fresher secondary phosphine solutions produced higher PL QYs, likely causing the PL QY ranges.

\begin{tabular}{|l|l|l|l|}
\hline $\begin{array}{l}\text { Secondary } \\
\text { phosphine used }\end{array}$ & PL QY (\%) range & PL QY (\%) average & PL FWHM (meV) \\
\hline DiBPSe & $40-86$ & 57 & 180 \\
\hline DPPSe & $12-18$ & 15 & 200 \\
\hline DEPSe & $8-18$ & 14 & 200 \\
\hline DiPPSe & $2-31$ & 13 & 140 \\
\hline DoTPSe & $2-9$ & 5 & 110 \\
\hline
\end{tabular}



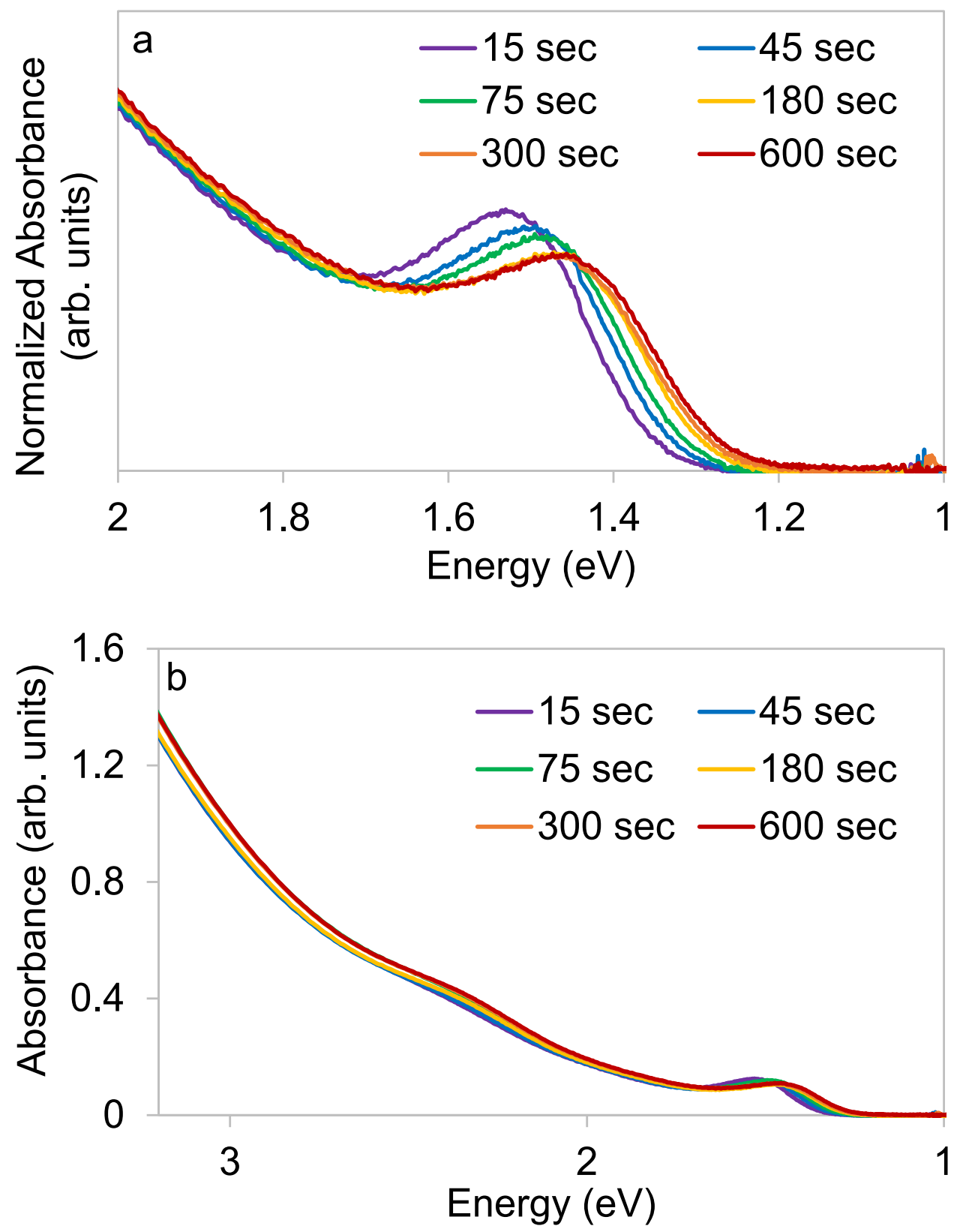

Figure S3. Representative absorption spectrum series, showing the reaction of DiBPSe with lead oleate. A ratio of 1:2.5:1 Se:Pb(OA) 2 :excess $\mathrm{OA}$ was used. Spectra were (a) normalized at 400 $\mathrm{nm}$ to correct for minor concentration differences, and (b) unnormalized. 


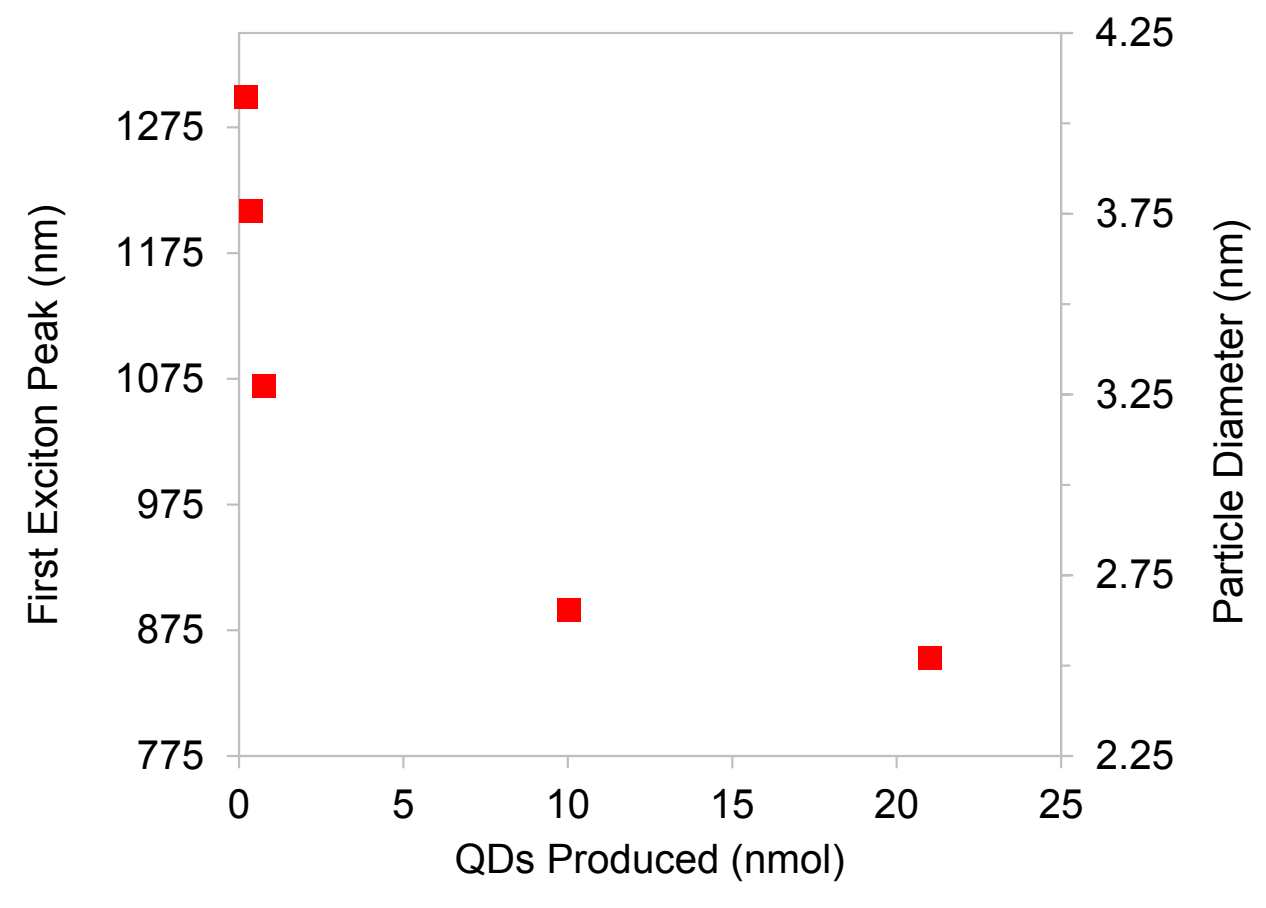

Figure S4. Under identical quantities of starting material, PbSe QD syntheses that produce small diameter nanocrystals result in a large number of QDs (for example, those made with DiBPSe), while syntheses that produce large diameter QDs (i.e., via DoTPSe) produce fewer QDs. 


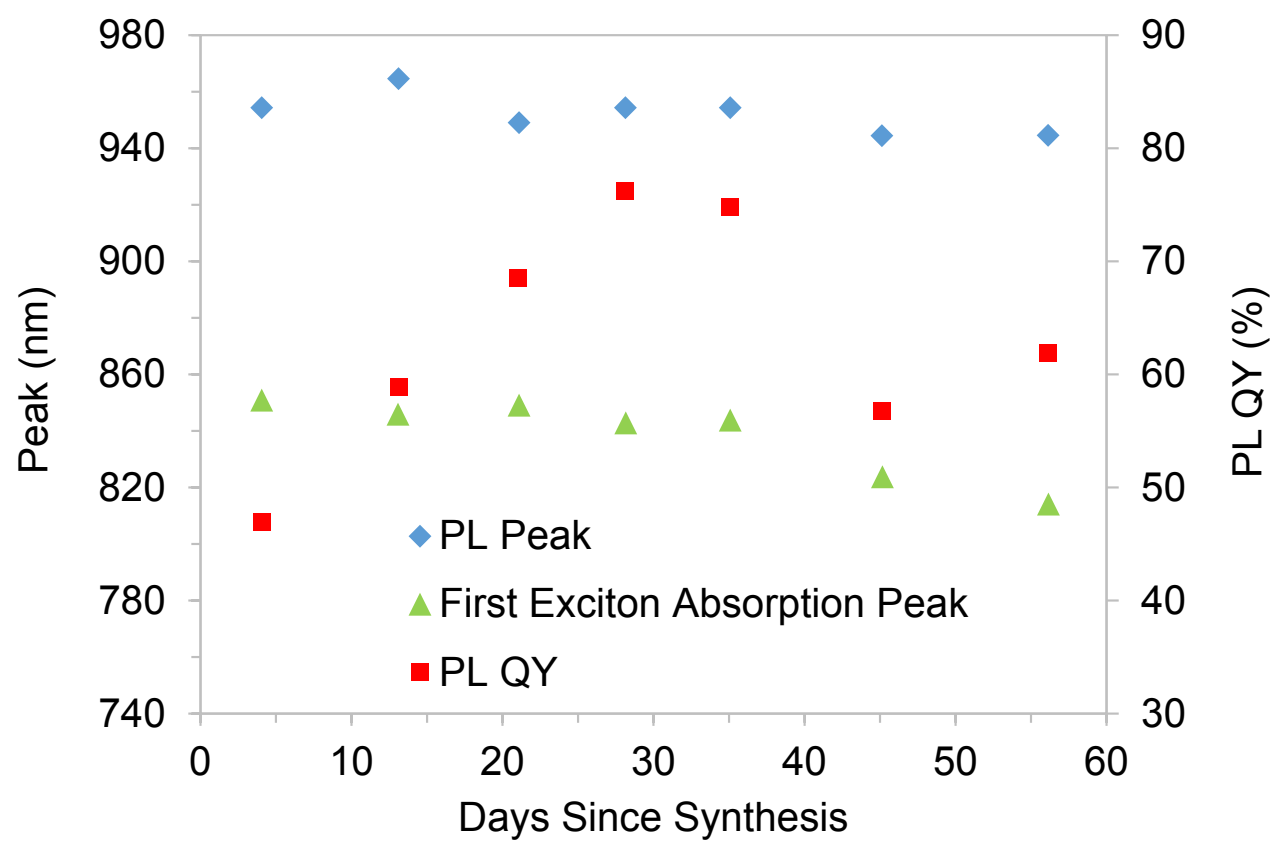

Figure S5. The PL QY, the wavelength of the first excitonic absorption peak, and the wavelength of the PL peak are monitored for a reaction of lead oleate and DiBPSe stored at $10^{\circ} \mathrm{C}$ over the course of eight weeks and demonstrates that these QDs are photostable. 


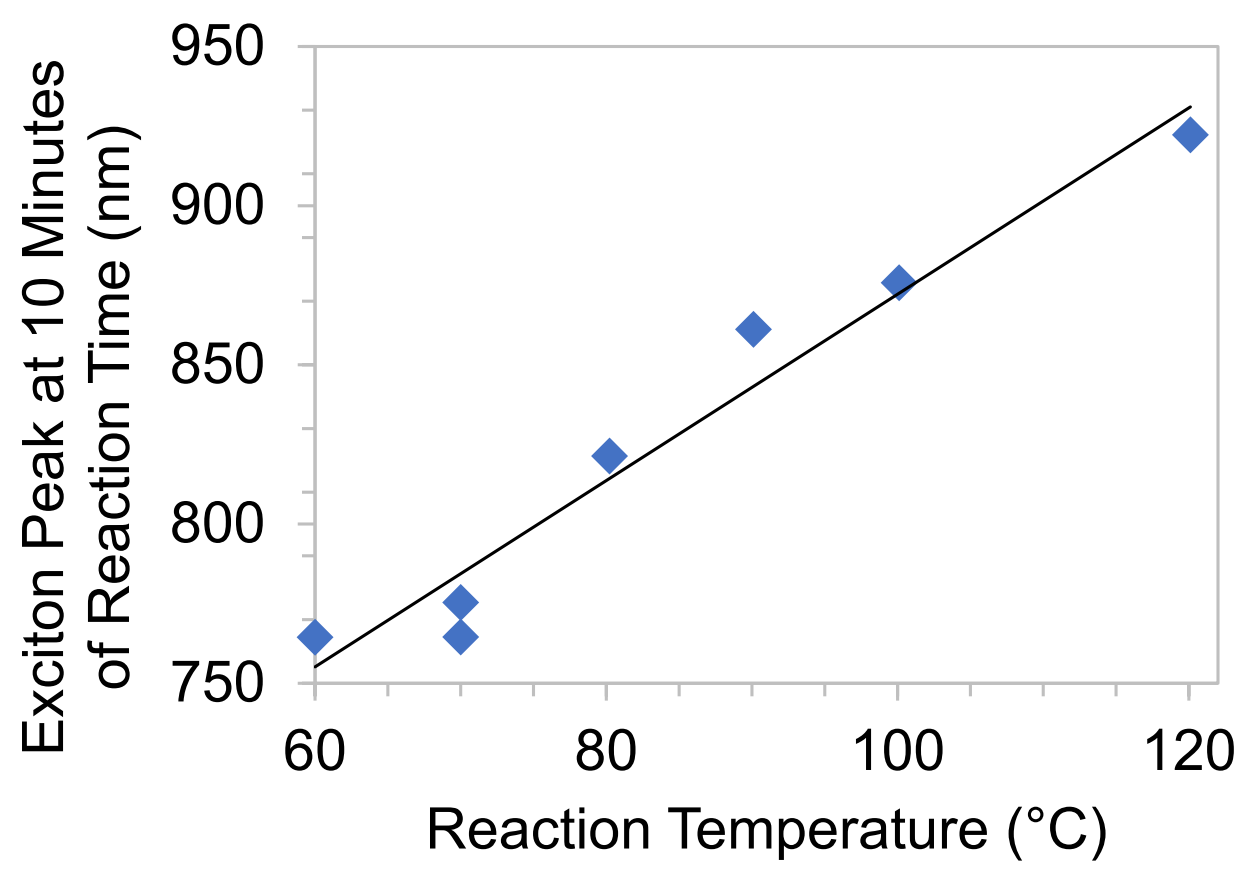

Figure S6. The wavelength dependence of the first excitonic peak of PbSe QDs produced from lead oleate and DiBPSe at varying temperatures. The line is a linear fit and can easily be used to produce QDs with a desired first exciton peak as given by: first exciton peak $(\mathrm{nm})=2.9 \times$ temperature $\left({ }^{\circ} \mathrm{C}\right)+579 \mathrm{~nm}$

Table S2. Reactions of lead oleate and DiBPSe at $100^{\circ} \mathrm{C}$ were conducted with varying precursor ratios. Ratios are presented as selenium to lead oleate to excess oleic acid in order to better demonstrate the effect of oleic acid in the system that isn't initially in the form of lead oleate. All QD sizes are calculated from the first excitonic absorption peak, according to literature. ${ }^{2}$

\begin{tabular}{|l|l|l|}
\hline $\begin{array}{c}\mathrm{Se}: \mathrm{Pb}(\mathrm{OA})_{2}: \text { excess OA } \\
\text { ratio }\end{array}$ & $\begin{array}{c}\text { Se:Pb:OA } \\
\text { ratio }\end{array}$ & \multicolumn{1}{|c|}{$\begin{array}{c}\text { QD Size Reached } \\
(\mathrm{nm})\end{array}$} \\
\hline $1.0: 1.5: 1.0$ & $1.0: 1.5: 4.0$ & 2.71 \\
\hline $1.0: 2.47: 1.0$ & $1.0: 2.47: 5.94$ & 2.58 \\
\hline $1.0: 5.0: 1.0$ & $1.0: 5.0: 11.0$ & 2.42 \\
\hline $1.0: 7.5: 1.0$ & $1.0: 7.5: 16.0$ & 2.43 \\
\hline $1.0: 10.0: 1.0$ & $1.0: 10.0: 21.0$ & 2.35 \\
\hline $1.0: 15.0: 1.0$ & $1.0: 15.0: 31.0$ & 2.32 \\
\hline $1.0: 2.47: 5.0$ & $1.0: 2.47: 9.94$ & 2.69 \\
\hline $1.0: 1.5: 10.0$ & $1.0: 1.5: 13.0$ & 3.19 \\
\hline $1.0: 2.47: 10.0$ & $1.0: 2.47: 14.94$ & 2.78 \\
\hline $1.0: 10.0: 10.0$ & $1.0: 10.0: 30.0$ & 2.37 \\
\hline $1.0: 10.0: 20.0$ & $1.0: 10.0: 40.0$ & 2.37 \\
\hline
\end{tabular}




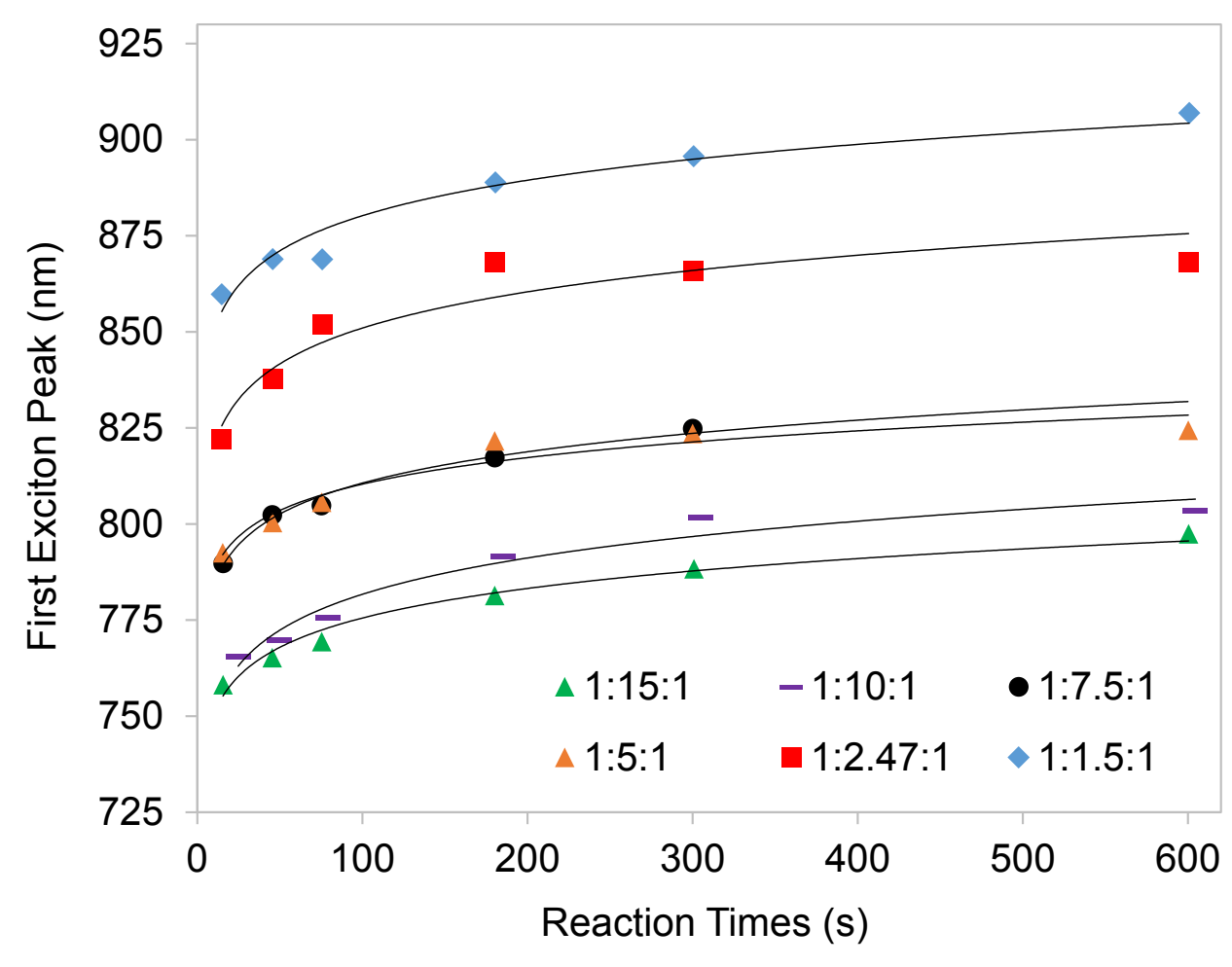

Figure S7. Reactions of lead oleate and DiBPSe where the lead oleate loading is altered demonstrates that higher lead loading results in smaller QDs. The figure legend indicates the ratio $\mathrm{Se}: \mathrm{Pb}(\mathrm{OA})_{2}$ : excess OA. 


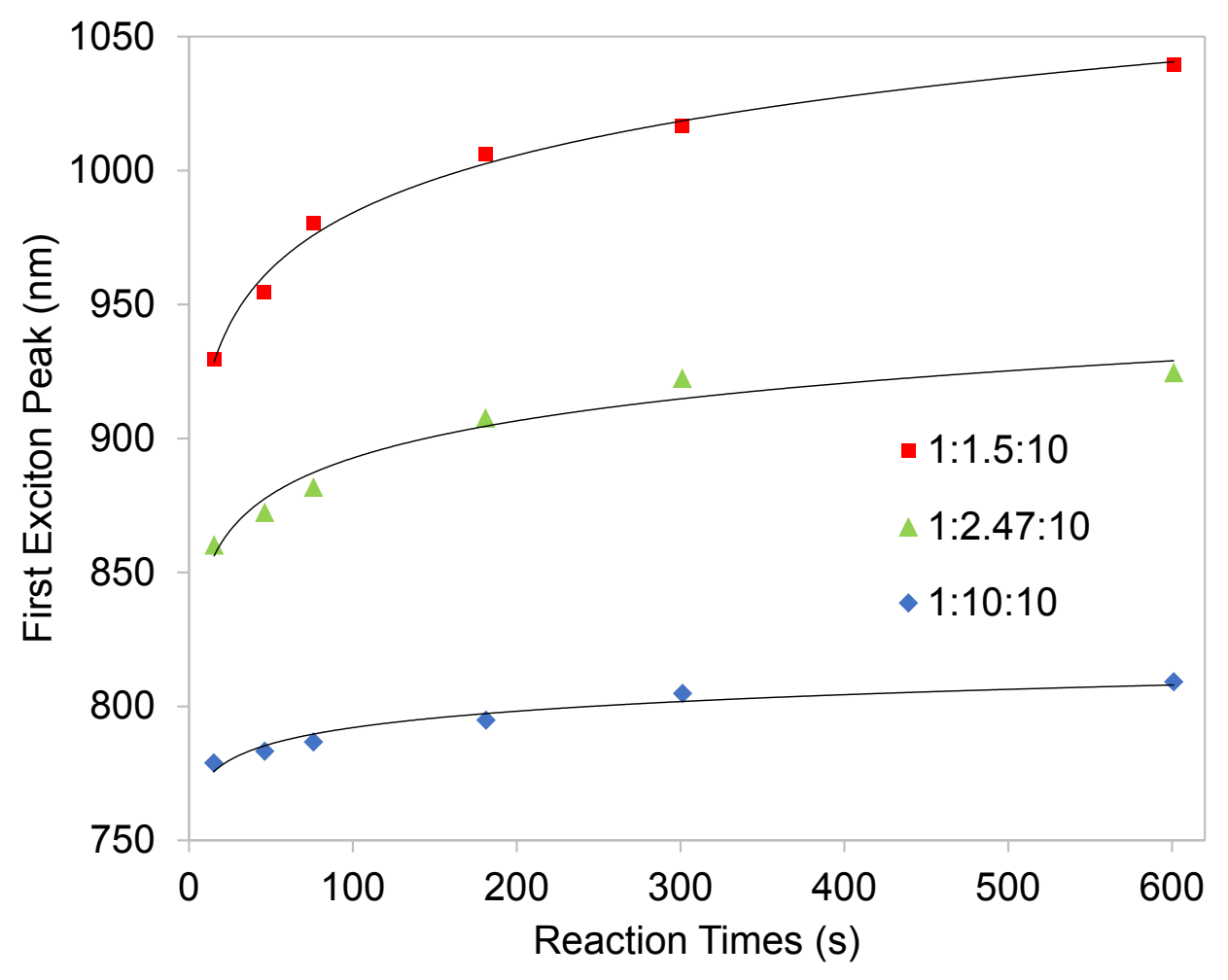

Figure S8. Reactions of lead oleate and DiBPSe where the lead oleate loading is altered under elevated, constant excess oleic acid, demonstrates that higher lead loading results in smaller QDs, but the additional oleic acid promotes larger growth than near-stoichiometric amounts of oleic acid. The figure legend indicates the ratio $\mathrm{Se}: \mathrm{Pb}(\mathrm{OA})_{2}$ : excess $\mathrm{OA}$. 


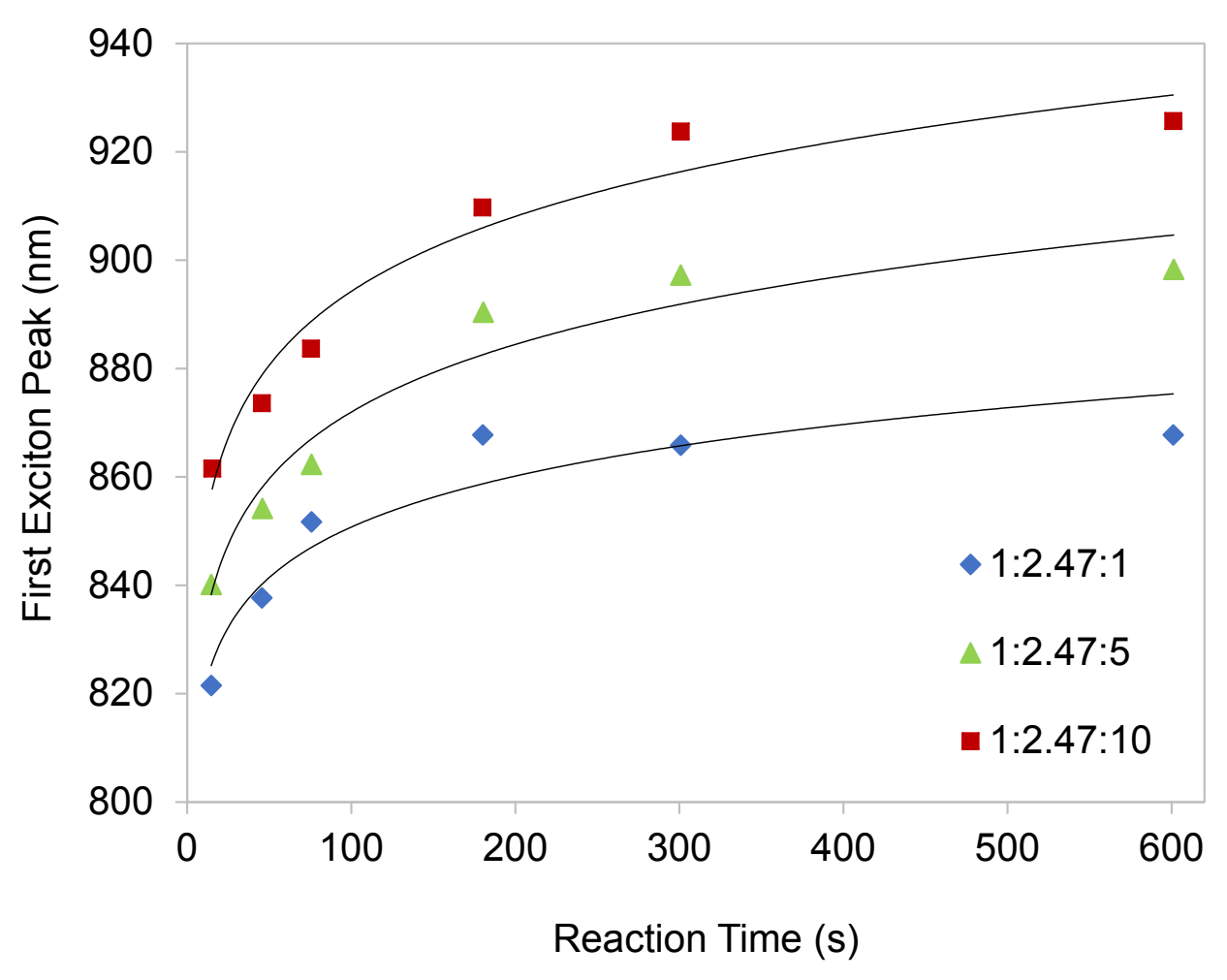

Figure S9. Reactions of lead oleate and DiBPSe where the excess oleic acid loading is altered demonstrates that higher quantities of free acid promotes larger growth (also corroborated in Figure S8). The figure legend indicates the ratio $\mathrm{Se}: \mathrm{Pb}(\mathrm{OA})_{2}$ : excess $\mathrm{OA}$. 


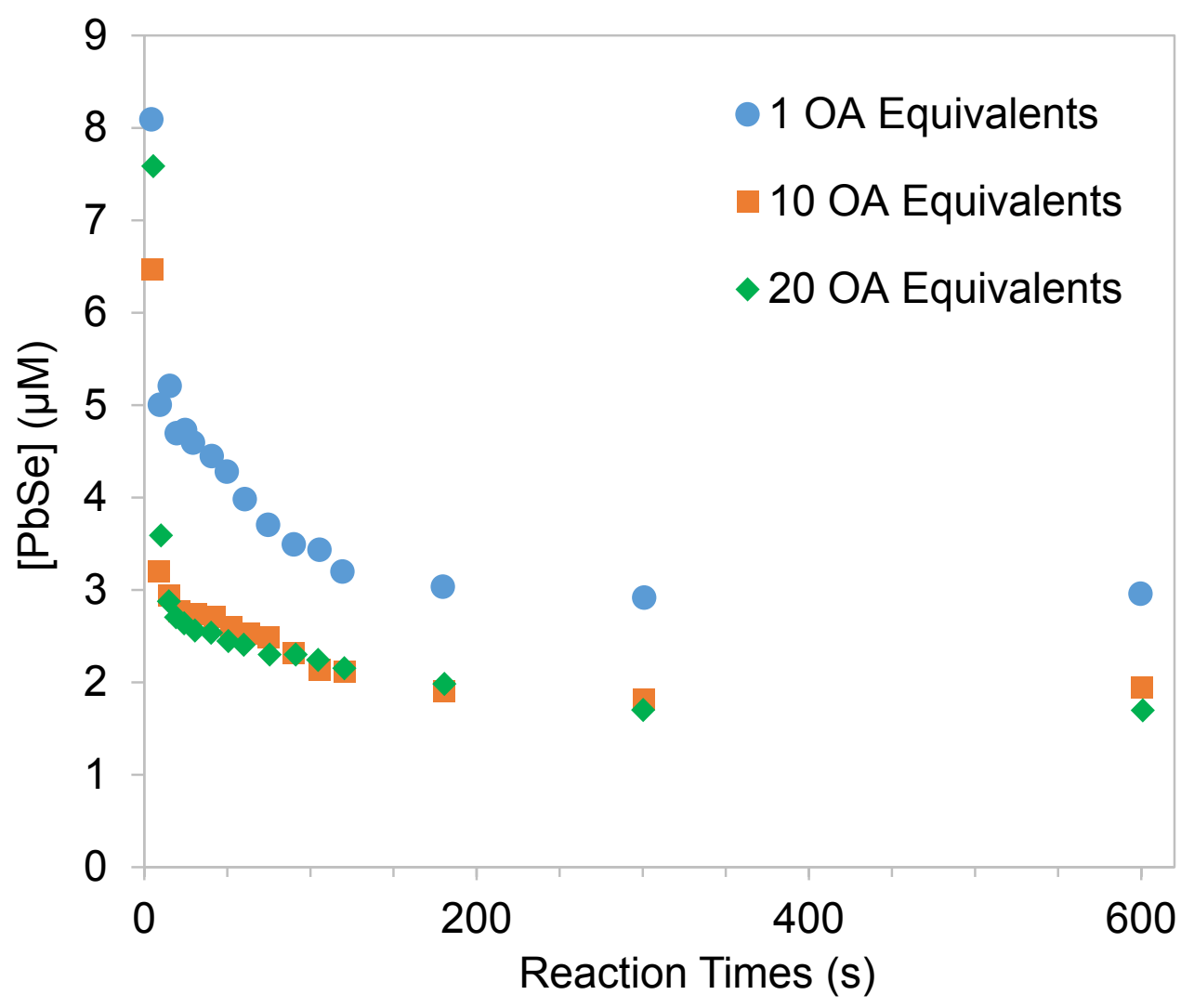

Figure S10. Reactions of lead oleate and DiBPSe where the excess oleic acid loading is altered demonstrates that higher quantities of free acid produces less QDs both initially and over the course of the reaction.

\section{Oleic Acid Purity}

The effect of oleic acid purity on QD kinetics was investigated by replacing $\mathrm{PbO}$ and $90 \%$ oleic acid with pre-synthesized purified lead olaeate $\left(\mathrm{Pb}(\mathrm{OA})_{2}\right)$ per an established method. ${ }^{3}$ In short, $2.5 \mathrm{~g} \mathrm{PbO}(99.999 \%$ purity) and $5 \mathrm{~mL}$ acetonitrile were placed in a $50 \mathrm{~mL}$ flask and stirred in an ice bath for at least 10 minutes. To this chilled solution was added $0.18 \mathrm{~mL}$ trifluoroacetic acid and $1.6 \mathrm{~mL}$ trifluoroacetic anhydride. This mixture was stirred on ice for 15 minutes, resulting in a clear and colorless solution. The mixture was then allowed to warm to room temperature. In a separate $250 \mathrm{~mL}$ roundbottom flask was mixed $6.359 \mathrm{~g}$ OA ( $99 \%$ purity), $45 \mathrm{~mL}$ isopropanol, and $2.562 \mathrm{~g}$ triethylamine. To this vessel was added the contents of the $50 \mathrm{~mL}$ flask while stirring, leading to the formation of a thick white precipitate and heavy white fumes in the flask headspace. This mixture was then heated to reflux, resulting in a clear, colorless solution.

After refluxing, the vessel was removed from heat to allow to slowly cool to room temperature for at least two hours. Then, it was placed in a $-4^{\circ} \mathrm{C}$ freezer for an additional two hours. A white mass resulted, which was washed under suction filtration using at least $225 \mathrm{~mL}$ methanol in a glass fritted funnel. After washing, the resulting white paste was dried under vacuum for at least six hours, and then transferred into a $\mathrm{N}_{2}$ glovebox for storage. 
Synthesis of PbSe QDs using high-purity oleic acid (99\% purity) followed the same synthesis technique as outlined in the Experimental section, with minor adjustments. In place of $\mathrm{PbO}$ and $90 \%$ oleic acid, $0.3851 \mathrm{~g} \mathrm{~Pb}(\mathrm{OA})_{2}(0.5 \mathrm{mmol})$ that was synthesized as described above was used. Further, an additional $0.0628 \mathrm{~g} \mathrm{OA}(99 \%)$ was added to ensure a final $\mathrm{Se}: \mathrm{Pb}(\mathrm{OA})_{2}$ :excess $\mathrm{OA}$ ratio of $1: 2.5: 1$.

Results of this synthesis can be seen in Figure S10. Use of high-purity OA resulted in QDs of slightly smaller mean diameter, with a maximum difference of $1.3 \AA\left(\Delta \mathrm{E}_{\text {peak }} \approx 70 \mathrm{meV}, \Delta \lambda_{\text {peak }} \approx\right.$ $40 \mathrm{~nm}$ ). As stated in the main document, the presence of oleic acid has the net effect of suppressing QD nucleation, thus producing overall larger QDs (and less QDs). We attribute the larger QD size when using lower-purity $\mathrm{OA}$ to the presence of impurities which affect the system by effectively slowing down the nucleation rate. Eliminating these impurities from the synthesis by using highpurity OA allows for increased nucleation rates and thus slightly smaller QD diameter.

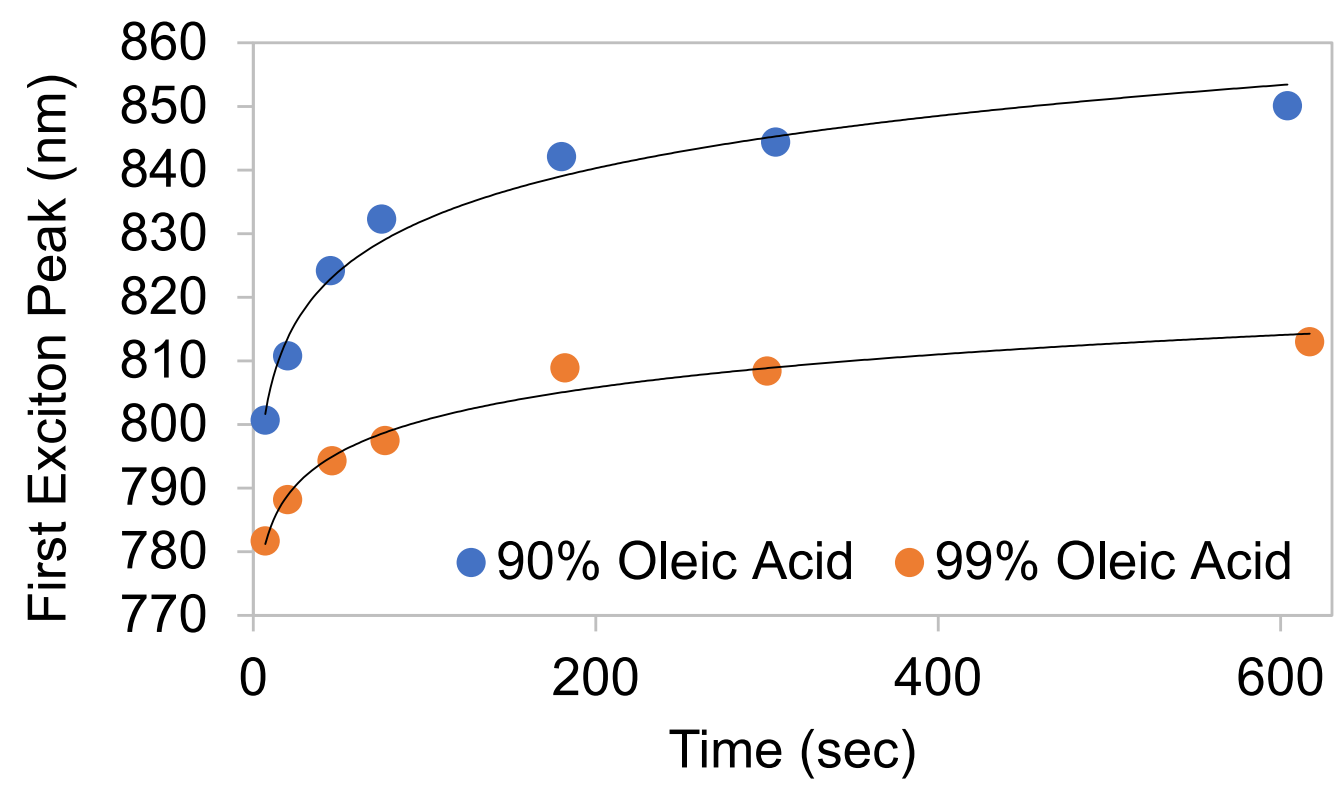

Figure S11. Reactions of lead oleate and DiBPSe where the oleic acid purity is altered demonstrates that higher purity results in slightly smaller QDs. The syntheses were carried out using a $\mathrm{Se}: \mathrm{Pb}(\mathrm{OA})_{2}$ :excess $\mathrm{OA}$ ratio of 1:2.5:1.

\section{References}

1. Dai, Q.; Wang, Y.; Li, X.; Zhang, Y.; Pellegrino, D. J.; Zhao, M.; Zou, B.; Seo, J.; Wang, Y.; Yu, W. W., Size-Dependent Composition and Molar Extinction Coefficient of PbSe Semiconductor Nanocrystals. ACS Nano 2009, 3, (6), 1518-1524.

2. Moreels, I.; Lambert, K.; Muynck, D. D.; Vanhaecke, F.; Poelman, D.; Martins, J. C.; Allan, G.; Hens, Z., Composition and Size-Dependent Extinction Coefficient of Colloidal PbSe Quantum Dots. Chem. Mater. 2007, (1), 6101-6106.

3. Campos, M. P.; Hendricks, M. P.; Beecher, A. N.; Walravens, W.; Swain, R. A.; Cleveland, G. T.; Hens, Z.; Sfeir, M. Y.; Owen, J. S., A Library of Selenourea Precursors to PbSe Nanocrystals with Size Distributions near the Homogeneous Limit. J. Am. Chem. Soc. 2017, 139, (6), 2296-2305. 
